\title{
¿Hablando se entiende la gente? Una propuesta práctica para desarrollar la interacción oral en la clase de lengua extranjera
}

\author{
MÓNICA BARROS LORENZO \\ Universidad de Oporto \\ mlorenzo@letras.up.pt \\ MARTA PAZOS ANIDO \\ Universidad de Oporto \\ manido@letras.up.pt
}

\begin{abstract}
Resumen: Con este trabajo se pretende reflexionar sobre la interacción oral en clase de lengua extranjera, sus protagonistas, cómo llevarla al aula y con qué objetivos. Asimismo, se considerarán algunas directrices para analizar y diseñar actividades y tareas de interacción oral que además respondan a las necesidades e intereses de nuestros estudiantes. Posteriormente, siguiendo las orientaciones anteriores, se desarrollará pormenorizadamente la propuesta práctica titulada «iCamarero, por favor!», dirigida a alumnos de nivel B1, cuyo objetivo principal es participar en una situación social -en este caso en un restaurante- (alumno como agente social) y actuar según las normas y convenciones sociales de la cultura meta (alumno como hablante intercultural), aplicando las estrategias y los procedimientos metacognitivos necesarios y reflexionando sobre su uso a lo largo del proceso de aprendizaje (alumno como aprendiente autónomo).
\end{abstract}

Palabras clave: actividad comunicativa de la lengua; actividades de interacción oral; interacción oral; conversación en el restaurante.

\section{Do we understand each other through dialogue? A practical proposal to develop oral interaction in foreign language classes}

\begin{abstract}
With this work we intend to reflect on the oral interaction in a foreign language class, its protagonists, how to include it to the classroom and with what objectives. Furthermore, some consideration will be given to guidelines to analyse and design spoken interaction activities and tasks that also respond to the needs and interests of our students. Subsequently, following the above guidelines, a practical proposal entitled "Waiter, please!", aimed at students of level B1, will be developed in detail. The main objectives here are for learners to participate in a social situation - in this case in a restaurant - (student as a social agent), to act according to the norms and social conventions of the target culture (student as an intercultural speaker), applying the necessary strategies and metacognitive procedures and to reflect on their use throughout the learning process (student as autonomous learner).
\end{abstract}

Keywords: communicative language activity; spoken interaction activities; spoken interaction; conversation in a restaurant. 


\section{Introducción}

La interacción oral (IO) constituye tanto para profesores como para alumnos una de las actividades comunicativas de la lengua más difíciles de desarrollar tanto dentro como fuera del aula (Pinilla Gómez, 2004: 890), por varias razones. Por un lado, esta actividad comunicativa de la lengua conlleva comprensión, interpretación y producción de mensajes y estos procesos pueden solaparse, pues en la interacción «procesamos, transmitimos, intercambiamos y negociamos información con uno o varios interlocutores» (2004a: 879). En la misma línea, el Marco común de referencia europeo para el aprendizaje, enseñanza y evaluación de lenguas (MCER) señala que en la IO «el usuario de la lengua actúa de forma alterna como hablante y oyente con uno o con más interlocutores para construir, conjuntamente, una conversación mediante la negociación de significados siguiendo el principio de cooperación» (Consejo de Europa, 2002: 74) y que «la interacción cara a cara puede suponer, naturalmente, una mezcla de medios: hablados, escritos, audiovisuales, paralingüísticos y paratextuales» (2002: 81).

En este sentido, en la comunicación entran en juego distintos aspectos que deben ser tomados en consideración por docentes y alumnos. De este modo, de acuerdo con los postulados de la lingüística sistémico-funcional defendidos por Halliday, se deberían tener en cuenta las funciones del lenguaje que condicionan la estructura del lenguaje: la ideacional, la interpersonal y la textual; así como el contexto situacional y el contexto cultural en el que toma sentido el texto (Halliday \& Hasan, 1989). En el primero intervienen las variables de campo (relacionado con el tema, con los significados experienciales del texto), tenor (con los participantes, su papel, su relación, sus actitudes, intenciones, etc.) y el modo (canal de comunicación) y estas variables conforman el registro, otra noción clave en esta teoría lingüística ligada a la adecuación al contexto (Halliday \& Hasan, 1989). Como defienden Fernández Colomer y Albelda Marco (2008):

Debemos conseguir que los estudiantes se puedan enfrentar a las diferentes situaciones y que adviertan sus diversas finalidades comunicativas. Si solo aprenden estructuras sintácticas, léxico y reglas gramaticales sin un contexto de uso y desvinculadas de sus funciones comunicativas, difícilmente podrán utilizar el idioma en las situaciones de la vida real. Reconocer la situación y adecuarse comunicativamente a ella es una exigencia para el que pretende desenvolverse en un idioma con eficacia (2008: 40).

Asimismo, se hace necesaria una reflexión sobre las características del discurso oral y del discurso escrito, dado que, como recomienda Pinilla Gómez: «[e]l usuario de lenguas extranjeras, sobre todo cuando ya ha alcanzado un cierto nivel debe ser consciente de las diferencias entre lo oral y lo escrito, para lograr un mejor dominio de ambos códigos» (2004: 883) a fin de que «pueda mejorar de modo general su competencia pragmática y, en particular, su competencia discursiva» (Llamas Saíz, 2006: 408). Estas dos modalidades discursivas no dependen únicamente, como advierte Llamas Saíz (2006), del modo o medio por el que se transmite un texto ni, como indican Fernández Colomer y Albelda Marco (2008), de su correspondencia con un tipo de registro, sino de varios factores pragmáticos, propiedades lingüísticas y factores contextuales, entre los que Llamas Saíz (2006: 403) destaca, adaptando una propuesta de González Ruiz y Martínez Pasamar: la espontaneidad, la copresencia (espacial y temporal) de los interlocutores, la relación entre ellos (de igualdad o jerárquica) y el saber compartido, los sentimientos y emociones del emisor, la dialoguicidad, la 
cooperación, la finalidad interpersonal de mantener un contacto (frente a la transmisión de información), la cotidianidad de los temas, etc.

Con relación a la espontaneidad es interesante observar cómo surgen las interacciones que se dan en el aula: de modo espontáneo (entre profesor y alumno o alumnos, entre alumnos, etc.) o planificadas previamente por el docente, pues esta variable conducirá a un tipo de discurso diferente. Algunos autores cuestionan la autenticidad de la lengua en las clases de idiomas, por ejemplo Widdowson (citado en Baralo Ottonello, 2000: 16) señala que «la autenticidad de la lengua en clase será siempre, hasta cierto punto e inevitablemente, una mera ilusión». Y en este sentido, afirma que dado que un aprendiente tiene en mayor o menor medida una competencia deficitaria de la lengua que está aprendiendo, no puede comportarse o dar las respuestas que daría un hablante de la lengua meta, de modo que su discurso no puede considerarse auténtico. Por ende, indica que entendiendo auténtico como sinónimo de natural, las interacciones orales en clase no son auténticas, sino artificiales, a pesar de que en ellas los estudiantes simulan o mantienen la ilusión de que están llevando a cabo interacciones naturales o espontáneas.

Sin embargo, otros autores no comparten esta opinión. Es el caso de Orta Gracia, quien diferencia entre interacción natural, interacción tradicional e interacción genuina en el aula, como puede verse en la siguiente figura (véase Figura 1), resaltando que «para que haya un aprendizaje significativo, debemos procurar que se establezca una interacción genuina y "auténtica" en el aula» (Orta Gracia, 2012: 5) y para promoverla podemos aprovechar los momentos de interacción espontánea que puedan surgir.

\begin{tabular}{|c|c|c|}
\hline Interacción natural & $\begin{array}{c}\text { Interacción tradicional } \\
\text { en el aula }\end{array}$ & $\begin{array}{c}\text { Interacción genuina en } \\
\text { el aula }\end{array}$ \\
\hline $\begin{array}{l}\text { Las personas deciden cuándo hablar, } \\
\text { dónde, con quién, de qué y cómo. }\end{array}$ & $\begin{array}{l}\text { El profesor decide el tema, } \\
\text { marca los turnos, exige un tipo } \\
\text { de producción formal. }\end{array}$ & $\begin{array}{l}\text { Se aprovechan las } \\
\text { oportunidades espontáneas de } \\
\text { comunicación. }\end{array}$ \\
\hline $\begin{array}{l}\text { En la vida buscamos, sobre todo, captar } \\
\text { la atención de nuestro interlocutor, } \\
\text { adecuando la forma al contenido y a los } \\
\text { efectos que deseamos. }\end{array}$ & $\begin{array}{l}\text { Los alumnos sólo se preocupan } \\
\text { de usar las estructuras } \\
\text { aprendidas sin importarles el } \\
\text { contenido y lo que } \\
\text { verdaderamente sienten. }\end{array}$ & $\begin{array}{l}\text { Los alumnos se centran en el } \\
\text { contenido y reflexionan sobre la } \\
\text { forma de su discurso para } \\
\text { mejorar y comunicar con mayor } \\
\text { precisión. }\end{array}$ \\
\hline
\end{tabular}

Figura 1: Interacción natural, tradicional y genuina en el aula (Adaptado de Orta Gracia, 2012: 5)

Por lo tanto, son muchos los aspectos que se deben contemplar a la hora de planificar una actividad ${ }^{1}$ de IO, así como las potenciales dificultades con las que nos podemos encontrar. Por consiguiente, en este artículo intentaremos abordar las cuestiones que se

\footnotetext{
${ }^{1}$ A fin de aclarar el uso de los conceptos de «tarea» y «actividad» tomaremos como referencia la descripción propuesta en el Diccionario de términos clave de ELE. Por actividad de aprendizaje entendemos «todas aquellas acciones que realiza el alumno como parte del proceso instructivo que sigue, ya sea en el aula de la lengua meta o en cualquier otro lugar (en casa, en un centro de autoaprendizaje, en un laboratorio de idiomas, etc.)» (Martín Peris, 1997-2016). Con frecuencia, el término se emplea como equivalente a tarea didáctica. En otras ocasiones, la actividad se entiende como un componente más de la tarea, como las fases preparatorias de la tarea final, junto con los objetivos, los contenidos, los materiales, etc. En este artículo optamos, por un lado, por el uso de «actividades», por ser extensivo a otros enfoques y seguimos la descripción anterior cuando presentemos la propuesta práctica; por otro, en la fundamentación teórica, al referirnos a los diferentes autores, respetaremos el concepto utilizado por ellos.
} 
deben considerar, las características de las actividades y las diferentes técnicas y estrategias que implementar con la intención de facilitar a nuestros alumnos el desarrollo de esta actividad comunicativa de la lengua, partiendo de que «la comunicación es un proceso, una "acción", que se concreta a través de una serie de habilidades que permiten su realización en una serie de "actos de habla" o intercambios comunicativos», tal y como apuntan las distintas aportaciones de disciplinas relacionadas con la didáctica de lenguas en el contexto de los enfoques orientados a la acción (Baralo Ottonello, 2000: 22).

\section{Aspectos importantes para planificar una actividad de interacción oral}

Como acabamos de comentar, la IO consiste en un proceso complejo que implica, como cualquier conversación real, «una sucesión de etapas que se desarrollan desde los intercambios iniciales hasta la conclusión» (Consejo de Europa, 2002: 122) que, como tal, requiere una serie de factores que se han de tener en cuenta en su planificación. A continuación, se señalan los que consideramos más representativos.

\subsection{El objetivo de la actividad}

Según Martín Peris (1999: 32), el objetivo puede hacer referencia a diferentes campos, como la comunicación lingüística, la forma lingüística, los contenidos temáticos y socioculturales y el aprendizaje de la lengua.

\subsection{El ámbito en que se contextualiza la actividad}

De acuerdo con la propuesta del $M C E R$, la actividad podría corresponder a los ámbitos público, privado, educativo y profesional (Consejo de Europa, 2002: 15).

\subsection{El papel del profesor y de los alumnos en la tarea}

En toda interacción son necesarios al menos dos interlocutores, por lo que es necesario decidir los actores de la comunicación y cuál es su papel, así como el tiempo de habla y los patrones de interacción, aspectos que también debe planificar el docente antes de la clase.

En lo que concierne al profesor, cuyo papel resulta fundamental, es aconsejable examinar su tiempo de habla (como señala González Argüello, 2015: 121: «[e]l profesor que mantiene interacciones equilibradas coloca al alumno en el centro del proceso de aprendizaje al darle oportunidades significativas de uso de la lengua»), el tiempo de espera dado a los estudiantes para responder a las preguntas planteadas, la zona de acción o las dinámicas y el tipo de preguntas que realizamos. Con respecto a estas últimas, conviene además observar cuáles prevalecen en nuestras clases (Richards \& Lockhart, 2008: 173-174): las procedimentales, relacionadas con las rutinas y procedimientos habituales en el aula (por ejemplo: ¿lo habéis entendido?, ¿está claro lo que tenéis que hacer?); las convergentes, cuya respuesta suele ser breve o de «sí» o «no», coincide normalmente con la buscada por el docente y no necesita de un alto grado de abstracción, crítica o análisis (por ejemplo: ¿qué pasó aquel día?, ¿quién estaba allí?); o las divergentes, caracterizadas por su carácter abierto, las cuales requieren un grado más alto de subjetividad, abstracción y reflexión que las convergentes y fomentan el intercambio de opiniones o indagan sobre diferentes interpretaciones de un hecho (por ejemplo: ¿qué pasaría si...?, ¿qué pensáis sobre...?). Así, como refiere González Argüello (2015: 121): «[s]i las preguntas que se le formulan en el aula exigen al alumno 
respuestas elaboradas, más posibilidades se le ofrecen de que pueda validar o refutar sus hipótesis sobre la lengua».

En el caso de los alumnos, al analizar sus participaciones orales también debemos tomar en consideración, entre otras cuestiones, su tiempo de habla, pues, de acuerdo con González Argüello (2015: 121), «[c]uanto mayor es el tiempo del que dispone el alumno para interactuar en el aula, mayores son las posibilidades de que pueda experimentar con el uso de la lengua»; las preguntas y respuestas que realizan o si la interacción es genuina. Asimismo, el enfoque elegido (normalmente los enfoques orientados a la acción implican una mayor participación del alumnado), los tipos de agrupamiento (individual, parejas, pequeños grupos o gran grupo) y los patrones de interacción -profesor-alumno(s), alumno(s)-profesor, alumno(s)-alumno(s)planificados o espontáneos pueden contribuir a aumentar el número de intercambios comunicativos.

Con el objeto de gestionar todos estos factores, debemos observar y estudiar nuestro comportamiento en el aula y las actuaciones del alumnado. En este sentido, podría resultar de utilidad el uso de plantillas de observación (Verdía Lleó, 2011:18) más o menos estructuradas focalizadas en el tipo de preguntas planteadas y su objetivo (activar conocimientos, evaluar, recordar contenidos vistos, repasar, reflexionar, invitar a participar, proporcionar retroalimentación, motivar, pedir opinión $\mathrm{u}$ obtener información, etc.), en el tiempo de habla del profesor y de los alumnos, en el tipo de interacción generada en cada caso (natural, tradicional o genuina), en los tipos de agrupamiento (cuáles consiguen incrementar la participación, motivación, establecimiento de relaciones personales, espíritu de cooperación y colaboración y sentido de responsabilidad del alumno) o en los patrones de interacción (cuáles son los más comunes, cómo y quién(es) toma(n) decisiones en torno a la interacción que se produce en el aula [inicio de la interacción, duración, tema, etc.] y con qué posibilidades se cuenta para desarrollarla). En este sentido, además de las fichas de observación, también podrían ayudar al profesor herramientas que fomentan la autorreflexión como el Portfolio Europeo para futuros profesores de idiomas ${ }^{2}$ (Newby et al., 2007).

\subsection{El contexto de la situación comunicativa}

De acuerdo con Pinilla Gómez (2004: 881), el contexto está integrado por el espacio, el tiempo y otras variables como el entorno social o cultural y la relación entre los participantes (edad, grado de conocimiento entre los interlocutores, etc.). Como ya se ha comentado cuando nos referimos a Halliday y Hasan (1989), con el fin de que la comunicación tenga éxito es necesario atender a estas circunstancias contextuales y las actividades propuestas deben promover la reflexión del alumno sobre estas cuestiones. Tampoco debemos olvidar la relevancia del contexto mental del hablante y de su interlocutor o interlocutores, pues el contexto externo en una interacción cara a cara es el mismo, pero la observación y la interpretación dependen del interlocutor. En cuanto a la percepción del mensaje, lo más difícil de superar son las diferencias de valores y creencias, de normas de cortesía, de expectativas sociales, etc., a no ser que los interlocutores ya hayan desarrollado una consciencia intercultural adecuada (Consejo de Europa, 2002: 54-55).

\footnotetext{
${ }^{2}$ En el caso concreto de la expresión e interacción orales conviene consultar el área «Hablar/Interacción oral», dentro de la categoría de «Metodología» (Newby et al., 2007: 21-22).
} 


\subsection{Los contenidos necesarios por parte del aprendiente}

Por contenidos entendemos no solo los lingüísticos, sino todos los relacionados con las cuatro competencias que, según la clasificación de Canale (1983), conforman la competencia comunicativa: la competencia lingüística, sociolingüística, discursiva y estratégica, así como la sociocultural y la social, añadidas por Van Ek (1986). En esta misma línea, cabe recordar la aclaración de Hymes (citado en Martín Peris, 1997-2016), quien a principios de los 70 , ya advierte de que la competencia comunicativa implica saber «cuándo hablar, cuándo no, y de qué hablar, con quién, cuándo, dónde y en qué forma»; es decir, no es suficiente con centrarse en el cómo se dice ni con preocuparse únicamente por la corrección lingüística.

Siguiendo con esta cuestión, conviene destacar que en el MCER (2002: 120) también se hace hincapié explícitamente en la importancia de las competencias pragmáticas que permiten al alumno organizar, estructurar y ordenar un mensaje (competencia discursiva), realizar las distintas funciones comunicativas (competencia funcional) y secuenciar los mensajes según esquemas de interacción y de transacción (competencia organizativa), competencias que deben desarrollar los estudiantes y que, consecuentemente, tienen que ser consideradas en la planificación de una actividad de IO. Asimismo, el MCER (2002: 87) alude expresamente a la comunicación no verbal, como las acciones paralingüísticas, el lenguaje corporal (gestos, expresiones faciales, posturas, contacto visual, contacto corporal, proxémica -tan habituales en las actividades orales cara a cara-), el uso de sonidos extralingüísticos en el habla (sonidos que implican significados que se han vuelto convencionales y reconocidos por la comunidad de habla, por ejemplo: chsss, agh, etc.) y las cualidades prosódicas (cualidad de voz, tono, volumen o duración).

\subsection{Las competencias y características de los alumnos}

Además de tomar en consideración los contenidos y los factores comunicativos, el docente debe planificar pensando en las competencias y características de los alumnos a los que se destina la tarea -los verdaderos protagonistas del proceso de enseñanzaaprendizaje- y determinar su potencial nivel de dificultad en función de estas variables. De este modo, prestará atención a:

- Los factores lingüísticos del alumno: el nivel y la fase de desarrollo en los que se encuentra (Consejo de Europa, 2002: 160).

- Los factores cognitivos: la familiaridad de la tarea (tipo, tema, género textual, pautas de interacción, etc.) y la capacidad de enfrentarse a ella (destrezas organizativas, interpersonales, estrategias de aprendizaje, interculturales, comprensión de las instrucciones, etc.) (Consejo de Europa, 2002: 158-159). Cabe señalar en este punto la propuesta de Nunan (1986: 142) de fijarse en lo que se espera que haga el alumno con la finalidad de determinar la dificultad de la tarea, como se observa en el siguiente esquema: 


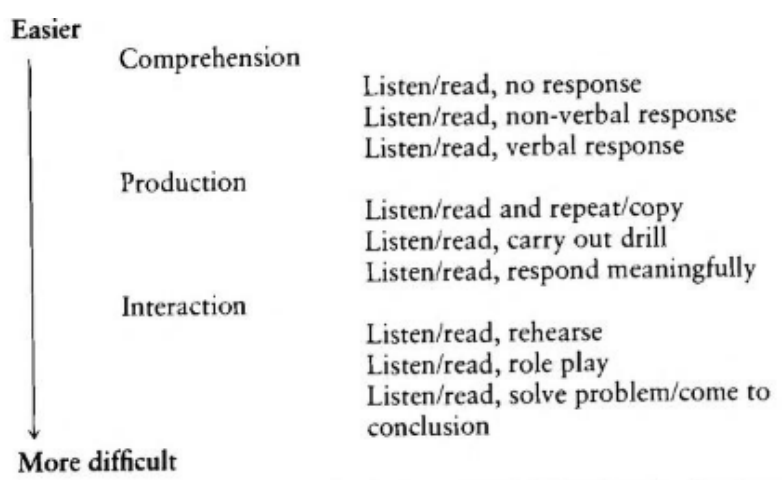

Figura 2: Determinación de la dificultad de la tarea según Nunan (1986: 142)

- Los factores afectivos: la autoestima, la implicación, la motivación, la inhibición, la actitud, etc. (Consejo de Europa, 2002: 159-160).

- Otros factores individuales relacionados con la personalidad, los valores, las creencias, los estilos cognitivos, etc.: «Los factores de actitud y de personalidad inciden enormemente no sólo en los papeles que cumplen los usuarios o alumnos de idiomas en los actos comunicativos, sino también en su capacidad de aprender» (2002: 104).

El papel fundamental que juega el factor afectivo en el proceso de enseñanzaaprendizaje no es algo nuevo, pues ya en los años 70 con las tendencias humanísticas en el campo educativo se reconocía su importancia (Arnold Morgan, 2015: 148) y hoy en día es aceptado por la mayoría del profesorado, aunque no siempre lo contemple a la hora de planificar. Stern lo deja claro en la siguiente cita: «the affective component contributes as least as much and often more to language learning than the cognitive skills» (citado en Arnold Morgan, 2015: 149), defendiendo así que la dimensión afectiva no se opone a la cognitiva, sino que ambas se complementan. Por esta razón, es primordial que los docentes trabajen los factores afectivos, traten de reducir el efecto de los negativos y estimular el de los positivos y creen un clima afectivo positivo en el grupo (Arnold Morgan, 2015) con la intención de que el aprendizaje en general sea más eficaz y, en particular, para las tareas de IO que normalmente generan aún más tensión en los alumnos. Sabemos que la autoestima, el autoconcepto, la seguridad en uno mismo y la falta de inhibición juegan a favor en la resolución de una tarea de IO, proporcionando al estudiante la confianza suficiente como para arriesgarse, probar, superar las dificultades y seguir intentándolo hasta lograr el objetivo establecido (Consejo de Europa, 2002: 159).

En este apartado dedicado a los factores del alumno que se deben analizar cuando se planifica una actividad de IO, se ha hecho referencia a la superación de las dificultades, lo cual nos lleva a pensar en el uso de estrategias. Por eso, en este punto hacemos un inciso con el fin de enfatizar su transcendencia, en este caso, las de IO para el desarrollo de la competencia comunicativa del aprendiente. Como ya apuntamos anteriormente, en nuestra lengua materna la interacción suele ser espontánea, automática, pero en la lengua extranjera no siempre ocurre lo mismo, por lo que conviene explicar a nuestros alumnos que, a pesar de que ya tienen esa capacidad, deben aplicarla de forma consciente a la lengua que están aprendiendo. En este sentido es necesario incluir actividades que potencien el entrenamiento estratégico de los estudiantes y que les 
ayuden a resolver los posibles problemas que surjan en sus interacciones. Ya hemos comentado que, según la concepción de competencia comunicativa de Canale (1983), la competencia estratégica es uno de sus componentes, la cual consiste en el uso de estrategias de aprendizaje y de comunicación, a las que el usuario de una lengua, ya sea nativa o extranjera, recurre cuando repara o compensa posibles dificultades comunicativas con el objeto de tener éxito en la interacción (Pinilla Gómez, 2004: 885886). En la oralidad, estas estrategias se utilizan tanto en los procesos de expresión como de recepción estando, por tanto, también implicadas en la interacción. Sin embargo, hay un tipo de estrategias exclusivas de la interacción que tienen que ver con el control de este proceso. A continuación, se señalan algunas de ellas:

\begin{tabular}{|c|c|c|}
\hline $\begin{array}{l}\text { Estrategias de } \\
\text { expresión oral }\end{array}$ & $\begin{array}{l}\text { Estrategias de } \\
\text { comprensión oral }\end{array}$ & Estrategias de interace \\
\hline $\begin{array}{l}\text { Transferencia y } \\
\text { aplicación de estrategias } \\
\text { de la lengua materna } \\
\text { - Verificación de } \\
\text { la comprensión correcta } \\
\text { - Suplencia de } \\
\text { carencias de expresión } \\
\text { (acuñación léxica, } \\
\text { recursos no verbales } \\
\text { como gestos o mímica, } \\
\text { paráfrasis, recursos a la } \\
\text { lengua materna, etc.) } \\
\text { Control de la } \\
\text { comunicación } \\
\text { (Pinilla Gómez, 2004b: } \\
439 \text { ) }\end{array}$ & $\begin{array}{l}\text { - Audición } \\
\text { selectiva de información } \\
\text { - Técnicas de } \\
\text { comprensión global } \\
\text { - Identificación } \\
\text { de los rasgos de } \\
\text { entonación } \\
\text { - Atención al al } \\
\text { contexto situacional }\end{array}$ & $\begin{array}{l}\text { PLANIFICACIÓN } \\
\text { - Encuadre (selección de praxeograma) } \\
\text { - Identificación de vacio de información y } \\
\text { de opinión } \\
\text { - Valoración de lo que se puede dar por } \\
\text { supuesto } \\
\text { - Planificación de los intercambios } \\
\text { EJECUCION } \\
\text { - Tomar la palabra } \\
\text { - Cooperación interpersonal } \\
\text { - Cooperación de pensamiento } \\
\text { - Enfrentarse a lo inesperado } \\
\text { - Petición de ayuda } \\
\text { EVALUACION } \\
\text { - Control de los esquemas y del } \\
\text { praxeograma } \\
\text { - Control del efecto y del éxito } \\
\text { CORRECCION } \\
\text { - Petición de aclaración } \\
\text { - Ofrecimiento de aclaración } \\
\text { - Reparación de la comunicación } \\
\quad \text { (Consejo de Europa, 2002: } 83-84 \text { ) }\end{array}$ \\
\hline
\end{tabular}

Figura 3. Estrategias de expresión, comprensión e interacción oral

\subsection{Otras condiciones y restricciones para la planificación de una actividad de IO}

Entre las condiciones y restricciones que se deben prever en la planificación de una tarea de IO para asegurar el éxito del alumno en su resolución, enumeramos las siguientes: el apoyo (el grado de contextualización que se facilita y la disponibilidad de ayuda lingüística con el objetivo de reducir la dificultad de la tarea), el tiempo (de preparación, de ejecución, la duración de los turnos de intervención y su duración total), la predicción ( $\mathrm{su}$ carácter predecible o impredecible, la introducción de nuevos elementos, etc.), las condiciones físicas (el ruido y las posibles interferencias) y los participantes (la colaboración del interlocutor o interlocutores, las características del habla -acento, ritmo, coherencia, etc.-, la visibilidad de los participantes -en las interacciones cara a cara los aspectos paralingüísticos que facilitan la comunicación- y las competencias generales y comunicativas de los interlocutores -conocimientos sobre 
el tema, comportamiento según las normas y convenciones de una comunidad concreta de habla-) (Consejo de Europa, 2002: 160-162).

\section{Características de una actividad de interacción oral}

A partir de la información de los párrafos anteriores intuimos algunas de las características que debería tener una actividad de IO para ser efectiva y afectiva. A continuación incidimos en algunas de ellas, en ocasiones, comunes también a otras actividades de recepción, producción y mediación:

- Integrarse con otras actividades comunicativas de la lengua. Pinilla Gómez explica que, como en la comunicación real, las distintas actividades comunicativas de la lengua han de aparecer integradas y no de forma aislada, a la vez que, como sugiere Candlin (citado en Estaire, 2009: 104), deben estar debidamente secuenciadas y organizadas:

Las actuales tendencias en metodología de la enseñanza de idiomas fomentan la realización de tareas comunicativas, en las cuales los alumnos aprenden una nueva lengua mediante un uso auténtico y efectivo de la misma, integrando de forma simultánea o sucesiva diversas destrezas en una misma actividad (Martín Peris, citado en Pinilla Gómez, 2004: 881-882).

- Centrarse en el significado. Garmendia Iglesias y Sans Baulenas comentan que «la enseñanza-aprendizaje de la lengua solo es eficaz si todas las actividades propuestas tienen en cuenta el significado» (2015: 96). Por un lado, se refieren a la significatividad de los textos, es decir, si son interesantes, representativos del mundo real, si les «dicen algo» a los alumnos; y, por otro, a la significatividad de su reacción al input presentado, o sea, si estos se enfrentan a los textos de manera natural y si se basan en el significado del input para reaccionar al mensaje (2015: 96-97).

- Ser comunicativa. Las actividades son comunicativas si «representan procesos de comunicación, y por tanto estimulan en el aprendiente la activación de esos procesos y, mediante ellos, el desarrollo de su competencia en la nueva lengua» (Martín Peris, 1999: 30). Asimismo, es importante valorar qué comunicación se está promoviendo, dado que a veces las actividades propuestas por los docentes conllevan la producción de enunciados que un usuario de la lengua competente no usaría cuando existe un contexto (Garmendia Iglesias \& Sans Baulenas, 2015: 97); por lo que la IO tendría que conducir a una producción lo más real y cercana posible a aquellas que el alumno realice fuera del aula (2015: 99). Por su parte, Orta Gracia sugiere realizar actividades en el aula que el estudiante también pueda hacer fuera y señala que «la vida fuera del aula es un excelente referente para negociar los objetivos de aprendizaje y diseñar la tipología de actividades» (2015: 85) y además aboga por aprovechar la espontaneidad que ofrece el aula para fomentar la interacción genuina, como ya apuntamos anteriormente.

- Ser abierta. Las actividades de IO han de permitir, como recomiendan Estaire (2004a) y Garmendia Iglesias y Sans Baulenas (2015: 99), que los alumnos puedan opinar, posicionarse, tener capacidad de elección y ser ellos mismos. En la misma línea, Orta Gracia (2015: 86) aconseja implicarlos en la planificación de actividades, por ejemplo, cediéndoles responsabilidades que tradicionalmente asume el profesor; y además, propone dejar espacio en la planificación a fin de 
que alumnos y docentes, puedan innovar y ser creativos, en lugar de planificar actividades poco flexibles o cerradas.

- Ser motivadora. Las actividades de IO deben fomentar la participación (trabajo cooperativo), crear interés y plantear vacíos de información, desafíos o problemas con la intención de promover la negociación entre todos los estudiantes y el espíritu crítico, de acuerdo con su nivel de desarrollo cognitivo y sus necesidades (Candlin, citado en Estaire, 2009: 114; Estaire, 2004a).

\section{Fases de una actividad de interacción oral}

Otra característica propia de una buena actividad de IO señalada por Estaire (2004b) es la estructura de la misma. Gelabert Navarro, Bueso Fernández y Benítez Pérez (2002: 39-40) proponen las siguientes fases para las actividades de producción oral: preactividad/es, en la(s) que se explica el motivo de su realización y se facilitan los contenidos necesarios para desarrollar la actividad (por ejemplo, actividades de motivación, de conocimiento del tema, de trabajo gramatical y léxico previo a la actividad, de avance de hipótesis sobre el contenido); la exposición clara del objetivo de la actividad, su organización y de los pasos a seguir para realizarla; el desarrollo de la actividad, durante la cual lo ideal es que el alumno participe y la intervención del docente sea mínima; la evaluación de la actividad, en la que se promueve la reflexión del alumno sobre su actuación y, si es necesario, se procede al tratamiento de posibles errores cometidos en la fase anterior; y la posactividad/es, en la que es posible ampliar la actividad realizada (por ejemplo, práctica y ampliación de los contenidos trabajados, trabajo de otras destrezas, juegos, etc.).

La secuenciación propuesta por estos autores permite establecer cierta correspondencia con las fases típicas del enfoque por tareas (Estaire, 2009): la pretarea, en la que se presenta la tarea, se explica (el contexto, el objetivo, el resultado esperado, los procedimientos que se van a seguir y el tiempo), se movilizan los conocimientos previos, se introducen los nuevos recursos necesarios y los alumnos planifican cómo van a realizar la tarea; la ejecución de la tarea que, en palabras de Estaire, «constituye el núcleo de la tarea» (2009: 89); y la postarea, en la que se fomenta una puesta en común para conocer los resultados o productos de la tarea, se promueve que el alumno reflexione sobre su propia actuación o la de su grupo y se pueden contemplar momentos de corrección o de ampliación de la práctica más centrados en la atención a la forma.

\section{Tipos de actividades}

Al catalogar las actividades de IO nos encontramos con múltiples tipologías, que en ocasiones presentan coincidencias, y que resultan pertinentes para la reflexión sobre cuáles nos interesa implementar en el aula. Entre ellas destacamos a modo de ejemplo:

- Conversaciones; preguntas y respuestas; resolución de problemas; debates, discusiones y argumentaciones; juegos comunicativos; simulaciones, dramatizaciones; chistes, relatos reales y fantásticos (Baralo Ottonello, 2000: 10).

- Transacciones, conversaciones casuales, discusiones formales, debates, entrevistas, negociaciones, planificaciones conjuntas, cooperaciones prácticas centradas en los objetivos, etc. (Consejo de Europa, 2002: 75).

- Encuestas o entrevistas, técnicas dramáticas (dramatizaciones, simulaciones y escenarios), exposiciones de temas, debates, conversaciones telefónicas y 
mensajes de contestador automáticos y actividades de carácter lúdico. (Pinilla Gómez, 2004: 891-893).

- Actividades de concienciación, actividades de práctica controlada y actividades de autonomía (Thornbury, 2005).

Para ayudar al docente a la hora de seleccionar, adaptar o planificar este tipo de actividades, puede resultar útil la propuesta del Plan Curricular del Instituto Cervantes $(P C I C)$, que presenta un inventario bastante completo y detallado de los contenidos de cada nivel establecidos por el MCER. Asimismo, dicho documento contempla un apartado dedicado a "Géneros discursivos y productos textuales» en el que se enumera un listado de géneros de transmisión oral en cada uno de los niveles, indicando además cuáles de ellos son de recepción y producción $(\mathrm{R})(\mathrm{P})$, solo de recepción $(\mathrm{R})$ o solo de producción $(\mathrm{P})^{3}$ que también orienta al docente en este proceso.

\section{Propuesta didáctica: «iCamarero, por favor!»}

En este punto pasamos a exponer la tarea que hemos titulado «iCamarero, por favor!» que, orientada a la acción y participación activa de los estudiantes, posee las características explicitadas anteriormente. Se trata, como se apunta en el PCIC, de una «conversación transaccional cara a cara» (2006: 332) que se presenta como una tarea final con varias actividades capacitadoras y una tarea derivada, que explicaremos a partir de las fases diferenciadas por Gelabert Navarro, Bueso Fernández y Benítez Pérez (2002: 39-40) ya referidas en este artículo. A continuación se facilitan más detalles sobre la propuesta:

Título: «¿Camarero, por favor!».

Nivel: B1 (adaptable).

Objetivo general: participar en una situación social, en este caso en una conversación cara a cara en un restaurante, actuando de acuerdo con las normas y convenciones sociales de la cultura meta, aplicando las estrategias y los procedimientos metacognitivos necesarios y reflexionando sobre su uso a lo largo del proceso de aprendizaje ${ }^{4}$.

\section{Contenidos ${ }^{5}$ :}

- Funciones: pedir la carta/el menú, solicitar información sobre un determinado plato, valorar un plato, ofrecer a otros, dar una orden de forma atenuada, llamar la atención de otro interlocutor para establecer la conversación de manera no verbal, pedir la cuenta, agradecer, repetir una orden o una información previa.

- Gramaticales: imperativo.

- Pronunciación: la entonación como exponente de los actos de habla orientados hacia el hablante (actos de habla exclamativos, expresivos) y hacia el oyente (actos de habla interrogativos e imperativos).

- Tácticas y estrategias pragmáticas: conversación transaccional en un restaurante, toma de iniciativa en la conversación, cortesía verbal atenuadora.

\footnotetext{
${ }^{3}$ Esta información puede consultarse en: http://cvc.cervantes.es/Ensenanza/biblioteca_ele/plan_curricular/niveles/07_generos_discursivos_introdu ccion.htm

${ }^{4}$ Para la formulación de este objetivo se han consultado los «Objetivos generales» del Plan Curricular del Instituto Cervantes (2006).

${ }^{5}$ Basados en los contenidos indicados en el Plan Curricular del Instituto Cervantes (2006).
} 
- Nociones generales: precios.

- Nociones específicas: alimentos, bebidas, platos, utensilios de cocina y mesa, menú del día, establecimientos.

- Referentes culturales: platos típicos.

- Saberes y comportamientos socioculturales: conceptos de menú del día, tapa, ración, pincho, aperitivo según la zona o región de la que se trate; comer de menú/a la carta; horarios de las comidas y de apertura de los bares, restaurantes, etc.; fórmulas utilizadas en la mesa; posibilidad de dejar propina; modalidades y formas de pago; formas de tratamiento al camarero.

- Habilidades y actitudes interculturales: curiosidad y apertura hacia las convenciones sociales y comportamientos de la cultura meta en relación con la situación comunicativa desarrollada en el restaurante (platos típicos, horarios, etc.).

- Procedimientos de aprendizaje: estrategias compensatorias (por ejemplo, gestos), estrategias de escucha activa.

Dinámicas: gran grupo (profesor-alumnos, alumno-alumno), pequeños grupos (alumnoalumno).

Materiales: tenedor $\mathrm{u}$ objeto similar para la actividad de motivación, grabaciones $\mathrm{y}$ transcripciones de conversaciones en bares y restaurantes, vídeo «Gestos» de Televisión española, menú seleccionado por los alumnos, papeles «secretos» para los alumnos que desempeñan el rol de clientes, ficha de planificación de la interacción oral (véase Figura 5).

\subsection{Pretarea}

Como actividad de motivación llevamos al aula un tenedor envuelto en papel burbuja con el objetivo de que a través del tacto los alumnos realicen hipótesis y traten de adivinar el objeto del que se trata, así como el tema en torno al que va a girar la tarea.

Una vez confirmadas las diferentes hipótesis, se propone a los estudiantes la realización de distintas comprensiones escritas y orales basadas en conversaciones en bares y restaurantes, en las que, de forma inductiva y a partir de varios diálogos tengan que analizar, como sugiere Esteve Ruescas (2016), el género textual del que se trata, la situación que representan, a los interlocutores y su relación, las actitudes de los hablantes, las características pragmáticas y los recursos gramaticales y semánticos necesarios para interactuar con éxito en este tipo de situaciones, etc. Así, tal como recomiendan Fernández Colomer y Albelda Marco: «[1]os textos reales y contextualizados ofrecen la ocasión de ejercitar una metodología inductivo-deductiva, donde los estudiantes inducirán las reglas y los fenómenos lingüísticos a partir de muestras de habla» $(2008$, p. 40$)$ y además «revelan gran cantidad de la cultura de una lengua concreta» (2008, p. 41). Entre estos ejemplos de conversaciones consideramos conveniente que aparezcan, por ejemplo, cómo pedir información sobre un determinado plato, valorarlo, y ofrecérselo a otros; cómo pedir la cuenta; cómo preguntar sobre las formas de pago; cómo dirigirnos apropiadamente a un camarero; cómo repetir una información cuando el otro interlocutor no escucha o no comprende (por ejemplo, debido al ruido que puede haber en bares y restaurantes); qué se entiende por menú del día en España y qué incluye; etc. ${ }^{6}$

\footnotetext{
${ }^{6}$ En este punto es aconsejable revisar otras convenciones sociales y comportamientos socioculturales relacionados con bares y restaurantes. Muchos de ellos aparecen indicados en el PCIC (2006: 409-410) y tienen que ver con cuestiones como las formas de pedir; los conceptos de tapa, ración, pincho, aperitivo
} 
Posteriormente, se trabajan también algunos elementos de la comunicación no verbal como la postura corporal, las expresiones de la cara, el contacto visual o los gestos, dada su importancia a la hora de comprender o confirmar lo que ocurre en las situaciones comunicativas cara a cara. Para ello, visionamos un sketch del programa de comedia de Televisión Española Splunge ${ }^{7}$ titulado $" \mathrm{Gestos}^{8}{ }^{8}$ en el que en un bar los clientes y la camarera terminan todas sus intervenciones con gestos cuyo significado es compartido por gran parte de la comunidad hispanohablante (como por ejemplo, los utilizados a la hora de pedir la cuenta, una caña o un café).

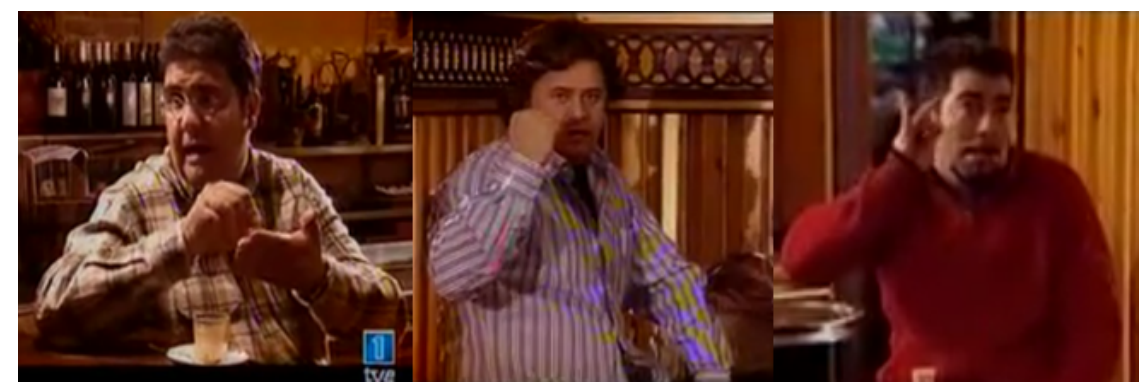

Figura 4. Fotogramas del programa Splunge de Televisión Española

Una vez revisados los puntos anteriores, se sugiere a los alumnos que, en grupos, busquen un menú de un restaurante español que será el que utilicen en la tarea. Posteriormente, deberán presentarlo brevemente al resto del grupo indicando el nombre del restaurante, la ciudad en la que se encuentra y el tipo de cocina que ofrece, a fin de que entre todo el grupo se elija uno de ellos.

\subsection{Objetivos y pasos de la tarea}

En esta fase se comunica a los estudiantes el objetivo de la tarea con mayor detalle (participar en una interacción social poniendo en juego sus conocimientos generales del mundo hispano en relación con los comportamientos en un restaurante utilizando vocabulario, estructuras, fórmulas y una gran variedad de funciones lingüísticas que les permita abordar esta situación comunicativa así como hablar de otros temas cotidianos durante la misma ${ }^{9}$ ), el tiempo previsto de realización de la tarea (aproximadamente veinte minutos para la planificación y quince para la interacción) y los pasos que van a seguirse para poder alcanzar el objetivo propuesto y que desarrollamos a continuación.

En primer lugar, se explica a los alumnos que primero trabajarán en pequeños grupos con otros compañeros que desempeñarán su mismo papel en la tarea: «camareros» o «clientes». Se recomienda que haya un «camarero» por cada tres o más grupos de «clientes» con la finalidad de que estos estén relativamente ocupados y no siempre disponibles, contribuyendo así a crear más espacios temporales en los que los

según la zona o región de la que se trate; la posibilidad de dejar propina; la variación de precios dependiendo de si nos sentamos en la barra, en la mesa o en la terraza; el carácter obligatorio de las listas de precios y libros de reclamaciones; los distintos modos de pago de consumiciones (fondo común, pagar por rondas, etc.); etc.

${ }^{7} \mathrm{https}: / /$ www.youtube.com/watch?v=IEGamVBeeOc

${ }^{8}$ Son muchas las propuestas didácticas para la enseñanza de ELE publicadas que utilizan este recurso audiovisual, por lo que convendría verificar si los alumnos ya conocen el vídeo antes de utilizarlo en clase.

${ }^{9}$ Adaptado de Instituto Cervantes (2006: 20-21). 
«comensales» se vean obligados a interactuar sobre otros temas de conversación. Los «camareros», además, se caracterizarán por tener algunos problemas de audición, por lo que «los clientes», con paciencia, tendrán que repetirles sus peticiones, así como recurrir a la comunicación no verbal para hacerse comprender.

En este momento de la planificación se proporcionan distintos recursos a «camareros»y «clientes» con la intención de que puedan realizar la tarea con éxito. Los primeros tendrán que analizar, con la ayuda del docente, el menú elegido previamente por todo el grupo y pensar en el esquema del posible intercambio comunicativo en el que van a participar. En el caso de los «clientes», estos deberán reflexionar sobre el praxeograma o esquema mental de la interacción o interacciones que se van a llevar a cabo (encuadre): de qué otros temas podrán hablar durante la comida y anticipar cuáles son las características de estas conversaciones (en función de si se trata de una comida con un grupo de amigos, familiares, compañeros de trabajo, etc.). Para ello, el profesor los guiará preguntándoles por ejemplo: ¿qué ocurre cuando estamos en una comida entre amigos, familiares, compañeros de trabajo, etc.? ¿Sobre qué hablamos: hechos recientes de nuestro pasado, anécdotas desconocidas para nuestra audiencia, etc.? ¿Tratamos los mismos temas en una comida con compañeros de trabajo o en una reunión de negocios? Asimismo, para fomentar la interacción se puede proporcionar distintos papeles «secretos» a cada uno de los «clientes»: por ejemplo, uno puede ser vegetariano, otro alérgico a las verduras o legumbres, otro diabético y otro tener que economizar por no disponer de mucho dinero en ese momento. Estos papeles, se reparten con el objetivo de que el menú tenga que leerse con más detenimiento, pues la elección de cada plato dependerá no solo de los gustos de cada «comensal», sino también de sus necesidades.

Con el fin de guiar y promover la reflexión sobre los aspectos antes citados, el docente facilitará a los alumnos una ficha similar a la que se presenta a continuación, adaptada del esquema SPEAKING propuesto por Hymes (citado en Pinilla Gómez, 2004: 881) ${ }^{10}$ :

\begin{tabular}{|l|l|}
\hline \multicolumn{2}{|c|}{ PLANIFICACION DE LA INTERACCION ORAL } \\
\hline Ambiente, escena: & \\
\hline Participantes: & \\
\hline Relación entre los participantes: & \\
\hline Características de los participantes: & \\
\hline Intención de las interacción/es: & \\
\hline Contenido: (lingüísticos, paralingüísticos, & \\
\hline $\begin{array}{l}\text { Recursos } \\
\text { pragmáticos, etc.) necesarios: }\end{array}$ & \\
\hline
\end{tabular}

Figura 5. Ficha para la planificación de una tarea de interacción oral a partir del esquema SPEAKING

\subsection{Realización de la tarea}

En este punto, se pone en práctica la planificación de la interacción descrita en la fase anterior, procediéndose a la realización simultánea de los diálogos por parte de los diferentes grupos ${ }^{11} \mathrm{y}$ a la posterior puesta en común.

${ }^{10}$ En este esquema, a través de una fórmula mnemotécnica se indican los elementos constitutivos de las distintas situaciones comunicativas: «Setting: ambiente, escena; Participantes; Ends: fines (intenciones como objetivos e intenciones como resultados); Art characteristics: forma del mensaje y contenido; Key: clave (fuerza ilocutiva) del mensaje y del tema; Instrumentalities: canal y códico; Normas de interpretación; y Género: tipo de acto de habla» (Pinilla Gómez, 2004: 881). 


\subsection{Evaluación}

En este momento tiene lugar el proceso de reflexión y evaluación sobre el desarrollo de la tarea (a nivel individual y grupal). Para ello, se solicita a los alumnos que expliquen, justifiquen o evalúen su desempeño analizando si han cumplido totalmente/en parte/nada los objetivos establecidos, los pasos llevados a cabo para su realización, etc. Puede resultar útil volver a la ficha de planificación de la IO presentada en el apartado $6.2 \mathrm{y}$ al análisis de los textos realizado en la actividad posibilitadora descrita en el apartado 6.1.

\subsection{Postarea}

En esta fase se pueden proponer diferentes actividades de ampliación y refuerzo de los pasos anteriores. Por ejemplo, una sugerencia es que los alumnos debatan sobre cuáles consideran las cinco reglas de oro para tener éxito como «clientes» o como «camareros» en un restaurante en España y que justifiquen sus decisiones. En caso de que no aparezcan entre las reglas propuestas aspectos relacionados con cómo es generalmente un menú del día, cómo se paga, cuándo se permite el tuteo o cuáles son los gestos con los que llamar al camarero o pedir la cuenta, convendría que el profesor fomentase esta reflexión.

\section{Reflexión final}

La enseñanza de la IO juega un papel decisivo en el proceso de enseñanza-aprendizaje de lenguas extranjeras y es, sin duda, una actividad compleja y difícil de llevar al aula. Además de necesitar una planificación de actividades bien secuenciadas que tengan en cuenta tanto las características propias de la comunicación oral como los intereses y necesidades de los aprendientes, requiere promover momentos de interacción genuina.

Asimismo, no hay que olvidar que los verdaderos protagonistas del proceso son los alumnos y que «a hablar se aprende hablando» y no solo analizando cómo se habla (aunque este sea un paso importante) (Montmany Molina, 2014), por lo que el aprendizaje de esta actividad comunicativa de la lengua conlleva práctica y consciencia de cómo funcionan los esquemas de interacción en distintas situaciones comunicativas contextualizadas. En este sentido, Esteve Ruescas (2016) recuerda también que el objetivo principal de los docentes no es que los alumnos construyan y representen interacciones correctamente, sino que aprendan a participar de forma adecuada en todo tipo de diálogos genuinos.

Para terminar y a modo de resumen coincidimos con Bygate, cuando plasma la repercusión de la enseñanza de la $\mathrm{IO}$, al referir que esta se convierte en muchas ocasiones en la «tarjeta de presentación» de nuestros estudiantes:

Speaking is in many ways an undervalued skill. [...] Speaking is, however, a skill which deserves attention every bit as much as literary skills, in both first and second languages. Our learners often need to be able to speak with confidence in order to carry out many of their most basic transactions. It is the skill by which they are most frequently judged, and through which they may make or lose friends. It is the vehicle par excellence of social solidarity, of social ranking, of professional advancement and of business. [...] Perhaps then, the teaching of speaking merits more thought. (Bygate, 1987: Introduction)

11 El profesor puede recomendar a los alumnos que graben sus interacciones con el objeto de que posteriormente puedan realizar una autoevaluación de su actuación. 


\section{Bibliografía}

ARnOld Morgan, J. (2015). «Explorando la dimensión afectiva de la enseñanza de ELE». En F. Herrera (ed.); La formación del profesorado de español. Innovación y reto (pp. 147-155). Barcelona: Difusión.

BARAlo OtTonello, M. (2000). «El desarrollo de la expresión oral en el aula de ELE», Carabela, 47, 5-36. Madrid: SGEL.

Bygate, M. (1987). Speaking. Oxford: Oxford University Press.

CANAle, M. (1983). De la competencia comunicativa a la pedagogía comunicativa del lenguaje. Antologías de textos de didáctica del español. Recuperado el 24/06/19, de: http://cvc.cervantes.es/ensenanza/biblioteca_ele/antologia_didactica/enfoque_comunicat ivo/canale01.htm

CONSEJO DE EUROPA (2002). Marco común de referencia europeo para las lenguas: aprendizaje, enseñanza y evaluación. Madrid: Instituto Cervantes, Ministerio de Educación, Cultura y Deporte y Editorial Anaya.

ESTAIRE, S. (2004a). «Esta tarea necesita una inyección», DidactiRed. Recuperado el 28/06/19, de: http://cvc.cervantes.es/aula/didactired/anteriores/marzo_04/29032004.htm

ESTAIRE, S. (2004b). «La programación de unidades didácticas a través de tareas», RedELE, 1. Recuperado el 28/06/19, de: http://www.mecd.gob.es/dctm/redele/MaterialRedEle/Revista/2004_01/2004_redELE_1_04Estaire.pdf?documentId=0901e72b80e068 11

ESTAIRE, S. (2009). El aprendizaje de lenguas mediante tareas: de la programación al aula. Madrid: Edinumen.

ESTEVE RuESCAS, O. (2016). «Replanteando el enfoque comunicativo en la enseñanza de lenguas en la universidad», Colóquio Internacional Aprolínguas. Porto: Faculdade de Letras da Universidade do Porto, 16-18 de junio de 2016.

Fernández Colomer, M. J. \& Albelda Marco, M. (2008). La enseñanza de la conversación coloquial. Madrid: Arco/Libros.

Garmendia Iglesias, A. \& SANS BAulenAs, N. (2015). «Diseño de materiales para la enseñanza y el aprendizaje de ELE». En F. Herrera (ed.); La formación del profesorado de español. Innovación y reto (pp. 91-100). Barcelona: Difusión.

Gelabert Navarro, M. J., Bueso Fernández, I. \& Benítez Pérez, P. (2002). Producción de materiales para la enseñanza de español. Madrid: Arco Libros.

GonZÁlez Argüello, M. V. (2015). «El discurso didáctico en el aula de ELE: ¿Sabemos cómo hablamos cuando hablamos en clase?». En F. Herrera (ed.); La formación del profesorado de español. Innovación y reto (pp. 115-123). Barcelona: Difusión.

Halliday, M.A.K. y Hasan, R. (1989). Language, Context and Text: Aspects of Language in a Social-Semiotic Perspective. Oxford: Oxford University Press.

Instituto Cervantes (2006). Plan curricular del Instituto Cervantes: niveles de referencia para el español. Madrid: Instituto Cervantes, Biblioteca Nueva. 
LLAMAS SAÍZ, C. (2006). «Discurso oral y discurso escrito: una propuesta para enseñar sus peculiaridades lingüísticas en el aula de ELE». En A. Álvarez et al. (eds.); La competencia pragmática y la enseñanza del español como lengua extranjera, (pp. 402411). Recuperado el 3/10/19, de: https://dialnet.unirioja.es/servlet/articulo?codigo $=2154313$

MARTín Peris, E. (1999). «Libros de texto y tareas». En J. Zanón (coord.); La enseñanza del español mediante tareas (pp. 25-52). Madrid: Edinumen.

MARTÍN PERIS, E. et. al. (1997-2016). Diccionario de términos clave de ELE. Recuperado el 29/06/19, de: http://cvc.cervantes.es/ensenanza/biblioteca_ele/diccio_ele/indice.htm

Montmany MolinA, B. (2014). «El aula como espacio de interacción». $5^{\text {as }}$ Jornadas didácticas de Difusión para profesores de ELE en Madrid. Madrid: Universidad Politécnica de Madrid, 21-22 febrero de 2014.

Newby, D., Allan, R., Fenner, A. B., Jones, B., Komorowska, H. \& Soghikyan, K. (2007). Portfolio Europeo para futuros profesores de idiomas. Una herramienta de reflexión para la formación de profesores. Graz: Consejo de Europa. Recuperado el 28/06/19, de: http://epost12.ecml.at/LinkClick.aspx?fileticket=Odz4pL2JvAk\%3D\&tabid=505\&langu age $=$ de-DE.

NunAn, D. (1986). Designing Tasks for the Communicative Classroom. Cambridge: Cambridge University Press.

ORTA GRACIA, A. (2012). «Las dinámicas para la interacción genuina en la clase de ELE». I Encuentro Práctico París. París. Recuperado el 28/06/19, de: http://www.encuentro-practico.com/paris/pdf/12/orta.pdf

ORTA GRACIA, A. (2015). «El arte de planificar y planificar con arte». En F. Herrera (ed.); La formación del profesorado de español. Innovación y reto (pp. 81-88). Barcelona: Difusión.

Pinilla Gómez, R. (2004). «La expresión oral». En J. Sánchez Lobato \& I. Santos Gargallo (dirs.); Vademécum para la formación de profesores. Enseñar español como segunda lengua (L2)/lengua extranjera (LE) (pp. 879-897). Madrid: SGEL.

RADIOTELEVISIÓN ESPAÑOLA (RTVE) [dronaleja]. (16 de junio de 2007). Splunge gestos [Archivo de vídeo]. Recuperado el 29/06/19, de: https://www.youtube.com/watch?v=IEGamVBeeOc

RICHARDS, J. C. \& LOCKHART, C. (2008). Estrategias de reflexión sobre la enseñanza de idiomas. Madrid: Edinumen.

THORnBury, S. (2005). How to teach speaking. Harlow: Pearson Education Limited.

VAN EK, J. A. (1986). Objectives for Foreign Language Learning (Vol I.). Strasbourg: Council of Europe.

VERDíA LLEÓ, E. (2011). «La observación de la actuación docente y la sesión de retroalimentación». Materiales del curso CFP228-10 La tutorización de las prácticas docentes de los profesores en formación. Formación de Profesores. Instituto Cervantes. 20-22 de junio 2011. 